\title{
Dental Occlusion Interpretation between Patient and Clinician in Eccentric Mandibular Positions
}

\author{
Nitikarn Ruttitivapanich ${ }^{1}$, Ratchawan Tansalarak², Jadesada Palasuk ${ }^{3}$, Thanaporn Sowithayasakul ${ }^{4}$ Jittima Pumklin $^{5}$
}

\begin{abstract}
Aim: This study aimed to compare between patient's and clinician's interpretations of dental occlusion in eccentric mandibular positions when T-Scan III was used as a standard device.

Materials and methods: Forty-three participants were trained to slide their jaw to the eccentric mandibular positions (right excursion, left excursion, and protrusion). The eccentric positions were determined at the edge-to-edge contact of anterior teeth in protrusion, the edge-toedge contact opposing canines, and/or contact of buccal cusps of the opposing premolars in the working position. Contacting pairs of teeth were recorded based on the patient's perception, clinician's interpretation using traditional occlusal indicators, and a digital occlusal indicator; T-Scan III. Weighted kappa was used to determine the correlation of dental occlusion obtained from the patient's perception and clinician's interpretation compared to T-Scan III.

Results: The highest correlation was in protrusion (substantial agreement; $\kappa_{\omega}=0.661$ for the patient group and 0.772 for clinician group) followed by left and right excursion (moderate agreement; the left excursion, $\kappa_{\omega}=0.569$ in both groups; the right excursion, $\kappa_{\omega}=0.430$ for the patient group and 0.545 for clinician group), respectively.

Conclusion: In a clinical situation, the interpretation of dental occlusion in eccentric mandibular positions acquired from a clinician is more dependable than information from patients.

Clinical significance: Dental occlusion evaluated by a clinician is more compatible with T-Scan III than that by patients.

Keywords: Dental occlusion, Eccentric position, Occlusal indicators, T-Scan.

World Journal of Dentistry (2021): 10.5005/jp-journals-10015-1798
\end{abstract}

\section{INTRODUCTION}

Clinically, the dental occlusion is routinely evaluated by both clinical examination and patient's perception. ${ }^{1}$ Patient's interpretation or perception comes from sensory afferent outputs from proprioceptors. ${ }^{2-4}$ Several investigators found that both vital teeth and pulpless teeth have a similar sensory response because they have the same periodontal mechanoreceptors (PMRs), not the sensory receptor from the pulp. This information indicated that PMRs which are essential in the perception of dental occlusion exist in both vital and non-vital teeth. ${ }^{2,4-6}$ They are located in the periodontal ligament (PDL) area and signal afferent outflow in response to the occlusal force when PDL is stretched. ${ }^{7-9}$

Occlusal indicators are conventionally used to examine occlusal contacts of opposing dentition. They are based on the coated ink marks of articulating papers on the tooth surface, removal force of shimstock foil, and perforation of occlusal indicator wax. These conventional techniques are straightforward but the outcomes vary due to the limited ability of waxes and color-coated paper to detect occlusal contact, clinician's experience, and the patient factors. To control these variations, computerized occlusal contact identifiers such as T-Scan have been advocated.

T-Scan III (Tekscan ${ }^{\circledast}$, Boston, MA, USA) is an example of the digital occlusal indicator. ${ }^{10,11}$ It can display dental occlusion as quantitative data in static and dynamic features. ${ }^{10-12}$ With the advantages of the quantitative information, T-Scan III is used in various fields in dentistry that require high accuracy. ${ }^{13,14}$ Although T-Scan III shows superior accuracy than the conventional techniques (i.e., using articulating paper or waxes), it is not routinely used in a clinical
${ }^{1}$ Dental Department, Photharam Hospital, Ratchaburi, Thailand

${ }^{2}$ Department of Preventive Dentistry, Faculty of Dentistry, Naresuan University, Phitsanulok, Thailand

${ }^{3-5}$ Department of Restorative Dentistry, Faculty of Dentistry, Naresuan University, Phitsanulok, Thailand

Corresponding Author: Jittima Pumklin, Department of Restorative Dentistry, Faculty of Dentistry, Naresuan University, Phitsanulok, Thailand, Phone: +66-89-683-6894, e-mail: Jittimap@nu.ac.th

How to cite this article: Ruttitivapanich N, Tansalarak R, Palasuk J, et al. Dental Occlusion Interpretation between Patient and Clinician in Eccentric Mandibular Positions. World J Dent 2021;12(1):7-11.

Source of support: The Graduate Students Research Funds, Faculty of Dentistry, Naresuan University

Conflict of interest: None

setting because of the high operating cost. Thus, the combined interpretation of dental occlusion achieved from patients and clinicians is still the key factor.

A previous study reported the association between T-Scan III and the correlation of bite force interpretation from patients and clinicians in maximal intercuspal position (MIP). Results showed that the bite force interpretation from the clinician is more clinically reliable than the patient's interpretation. ${ }^{15}$ Currently, a study of dental occlusion in eccentric mandibular positions has not been investigated. Therefore, this study aimed to compare the interpretation of dental occlusion between patients and a clinician in eccentric mandibular positions when T-Scan III was served as a standard device. 


\section{Materials and Methods}

Naresuan University Ethical Committee approved the study protocol with an IRB No. 0995/60 before data gathering. The undergraduate dental students at Naresuan University (Phitsanulok, Thailand) participated in this study because they have clinical experience, understand tooth contact patterns (i.e., mandibular posture), and no communication problem during data collection.

Sample size justification was calculated using GPower 3.1 ${ }^{\oplus}$. The total sample would be 100 for three independent variables (patient's perception, clinician examination, and T-Scan III analysis). To compensate for the sample loss (approximately 10-20\%), the total sample size would be 110 to 120 . The dental occlusion of each participant was required to be examined by all three independent variables. Therefore, the sample size of the current study was set to have at least 40 samples $(n=40)$. Finally, 43 participants took part in this study following the inclusion and exclusion criteria as follows:

\section{Inclusion Criteria}

Class I molar relationship according to Angle's classification. Positive vertical and horizontal tooth overlap.

Minimum of 6 teeth/quadrant (12 teeth/arch) with the contralateral teeth.

\section{Exclusion Criteria}

Tooth mobility (2nd to 3rd degree).

Pending active orthodontic treatment.

Temporomandibular disorder.

Parafunctional habit.

Ongoing dental treatment during the period of the study.

\section{Methods}

All participants were repeatedly trained to move their mandible to the eccentric mandibular positions (right excursion, left excursion, and protrusion) before data collection. The working excursion is determined at the edge-to-edge contact of the opposing canines and/or contact of buccal cusps of the opposing premolars. For the protrusion, it is at the edge-to-edge contact of anterior teeth.

Two appointments were desired for each participant. For the first appointment, alginate impressions were made, and the mesiodistal width of teeth was measured from the study models to set the T-Scan III program. Then, an occlusion specialized dentist (Jittima Pumklin; JP) examined the dental occlusion using articulating papers (Hanel ${ }^{\oplus}$, Langenau, Germany) and shimstock foils (Hanel ${ }^{\oplus}$, Langenau, Germany) to test intra-examiner reliability.

The second appointment was done a week later. Evaluation of dental occlusion was acquired by means of patient's perception of their tooth contacts, a clinical examination from the clinician (JP), and T-Scan III analysis. Firstly, participants were asked to bite on MIP and move the mandible to eccentric mandibular positions (right excursion, left excursion, and protrusion). Then, patients reported contacting teeth through their index fingers. Each recording position was performed in triplicate. Secondly, JP evaluated and clarified contacting teeth during eccentric mandibular position using articulating papers (Hanel ${ }^{\circledR}$, Langenau, Germany) and shimstock foils (Hanel ${ }^{\circledR}$, Langenau, Germany). Lastly, T-Scan III data were collected by asking participants to bite on the T-Scan III HD sensor at the recording positions. The contacting teeth (blue mark) were shown in the tooth diagram (Fig. 1). T-Scan III analysis was also repeated three times.
The contacting teeth during the eccentric mandibular positions were recorded and classified as "anterior", "posterior", or "anterior and posterior" for the right and left excursions. The recording data were classified as "right", "left", or "right and left" for the protrusion. These data indicated which part/side of the jaws guided the eccentric movement regardless of the number of tooth contacts.

Intra-examiner reliability (JP) of clinician interpretation was tested by stratified random sampling from 16 participants during both appointments. Cohen's weighted kappa was calculated (SPSS 17.0, Inc., Chicago, IL, USA) to determine the intra-examiner reliability [kappa score $\left(\kappa_{\omega}\right)$ ] and the correlation of dental occlusion compared to T-Scan III (a standard device). Intra-examiner reliability was classified as shown in Table $1 .^{16}$

\section{RESULTS}

Forty-three participants consisted of 29 females and 14 males. Their age ranged from 19 to 23 years old. The tooth contact agreement between the patient's perception in eccentric movement and T-Scan III were $65.12 \%$ (right excursion), $72.09 \%$ (left excursion), and $79.07 \%$ (protrusion) (Table 2). The tooth contact agreement between clinician interpretation in eccentric movement and T-Scan III were $72.09 \%$ (left and right excursion) and $86.05 \%$ (protrusion) (Table 3).

Weighted kappa analysis revealed that the protrusion had the best agreement (highest kappa score) with T-Scan III among three eccentric movements in both patient and clinician interpretation (substantial agreement; $\kappa_{\omega}=0.661$ for the patient and $\kappa_{\omega}=0.772$ for the clinician).

The moderate agreement with T-Scan III of patient and clinician interpretation was observed for lateral excursions. Kappa scores of the right excursion were 0.430 for patient interpretation and 0.545 for clinician interpretation. Kappa scores of the left excursion were 0.569 for both groups (Table 4 and Fig. 2). Intra-examiner clinician reliability (JP) was moderate to an almost perfect agreement (Table 5).

\section{Discussion}

Although the digital occlusal indicators offer superiority over the traditional occlusal indicators, the high cost of the equipment is

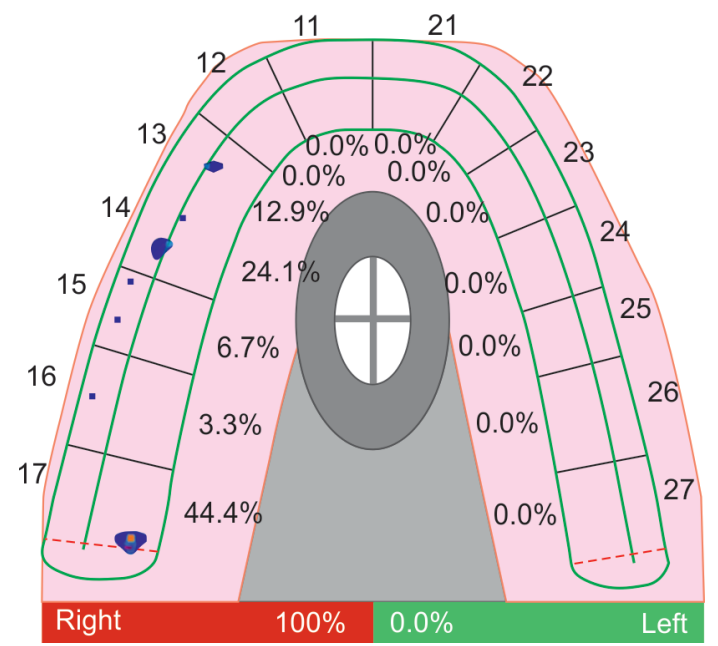

Fig. 1: Tooth diagram from T-Scan III showed the contacting teeth (blue mark) during the right excursion 
the main reason why they are not widely used. Currently, patients and clinicians are still the keys to detect dental occlusion. A previous study showed that the bite force interpretation in MIP from clinicians is more clinically reliable than patients. ${ }^{15}$ No study has ever assessed the interpretation of the dental occlusion in an eccentric position.

This is the first study to have evaluated the dental occlusion interpretation between patients and a clinician in eccentric mandibular positions. The results showed that the highest agreement (substantial agreement) with T-Scan III was protrusion for both patient recognition and clinician interpretation/examination, followed by moderate agreement on the left and right excursions, respectively. Even though both patient recognition and clinician interpretation had the same agreement with T-Scan III, clinician interpretation showed a higher percent of subject agreement than patient's perception. This finding is possibly due to the inability of patients to discriminate the light contact between the opposing teeth. Siirilä and Laine found that humans can discriminate approximately $8-10 \mu \mathrm{m}$ of foreign bodies. ${ }^{17}$ Their results suggested that humans cannot detect tooth contacts that are $<8-10 \mu \mathrm{m}$. On the other hand, if the interocclusal space between the occluding teeth is $>8-10 \mu \mathrm{m}$, humans can pinpoint the contacting pairs of teeth or identify which side of jaws contacts heavier.

In protrusion, incisors guided the protrusive movement as the incisal edges of mandibular incisors moved along the lingually inclined planes of maxillary incisors. ${ }^{18}$ The contacting teeth in protrusion are often located on anterior teeth. Anterior teeth have highly sensitive PMRs to force magnitudes than the posterior teeth have. ${ }^{5,8,19}$ Additionally, studies reported that the most sensitive area to bite force perception is at incisor, canine, and premolar regions,

Table 1: Categorized Cohen's weighted kappa ${ }^{16}$

\begin{tabular}{ll}
\hline Kappa score & Interpretation (agreement level) \\
\hline$<0$ & Poor \\
$0.00-0.20$ & Slight \\
$0.21-0.40$ & Fair \\
$0.41-0.60$ & Moderate \\
$0.61-0.80$ & Substantial \\
$0.81-1.00$ & Almost perfect \\
\hline
\end{tabular}

Table 2: The contacts agreement of patient's perception compared to T-Scan III analysis in eccentric positions

\begin{tabular}{lll}
\hline & \multicolumn{2}{c}{ Subject agreement (total number $=43$ ) } \\
\cline { 2 - 3 } Eccentric position & Number & Percent \\
\hline Right excursion & 28 & 65.12 \\
Left excursion & 31 & 72.09 \\
Protrusion & 34 & 79.07 \\
\hline
\end{tabular}

Table 3: The contacts agreement between clinician interpretation and T-Scan III analysis in eccentric positions

\begin{tabular}{lll}
\hline & \multicolumn{2}{c}{ Subject agreement (total number $=43$ ) } \\
\cline { 2 - 3 } Eccentric position & Number & Percent \\
\hline Right excursion & 31 & 72.09 \\
Left excursion & 31 & 72.09 \\
Protrusion & 37 & 86.05 \\
\hline
\end{tabular}

respectively. ${ }^{20,21}$ Johnsen et al. compared the periodontal afferents from the different areas of the dental arch by peanut holding. They found that the more distal part of the dental arch, the more bite force generated. Their finding supported the high sensitivity of PMRs in the anterior teeth, rather than the posterior teeth. ${ }^{20}$ Therefore, in the current study, the highest agreement with T-Scan III in protrusion compared to other movements can be explained by the highly sensitive PMRs in the anterior region resulting in an increased ability of participants to identify tooth contacts in the anterior teeth than the posterior teeth.

In lateral excursion, the mandible was guided to move away from MIP along the palatal inclined plane of maxillary canine and/ or palatal inclined planes of buccal cusps of maxillary posterior teeth. ${ }^{22,23}$ Several investigators reported that the most common lateral excursive pattern in young adults is a group function occlusion. ${ }^{24-27}$ In this study, posterior teeth (not the canine) which had poorer PMRs than the anterior teeth ${ }^{20}$ were mainly guided the mandible during the lateral excursion. Therefore, the similar correlation between left and right excursion (moderate agreement), and the lower correlation to T-Scan III than protrusion (a substantial agreement, guided by anterior teeth) may be explained by the poorer PMRs in the posterior segment of the jaw. Moreover, locating the contacting teeth in the posterior segment of the jaw is more difficult than the anterior region (i.e., in protrusion) because they are on the side of the jaw which is not easily seen with the patient's eyes, and contacting teeth are usually obscured by the surrounding tissue (i.e., cheek).

For the standard device, the T-Scan III system was used in this study because this system can report quantitative data, whereas traditional occlusal indicators cannot. Additionally, the HD sensor

Table 4: Cohen's weighted kappa between patient's perception, clinician interpretation, and T-Scan III analysis at eccentric positions

\begin{tabular}{lllll}
\hline & \multicolumn{2}{c}{ Patient's perception } & \multicolumn{2}{c}{ Clinician interpretation } \\
\cline { 2 - 5 } & Kappa & Agreement & Kappa & $\begin{array}{l}\text { Agreement } \\
\text { level }\end{array}$ \\
\hline Eccentric positions & score & level & score & level \\
\hline Right excursion & 0.430 & Moderate & 0.545 & Moderate \\
Left excursion & 0.569 & Moderate & 0.569 & Moderate \\
Protrusion & 0.661 & Substantial & 0.772 & Substantial \\
\hline
\end{tabular}

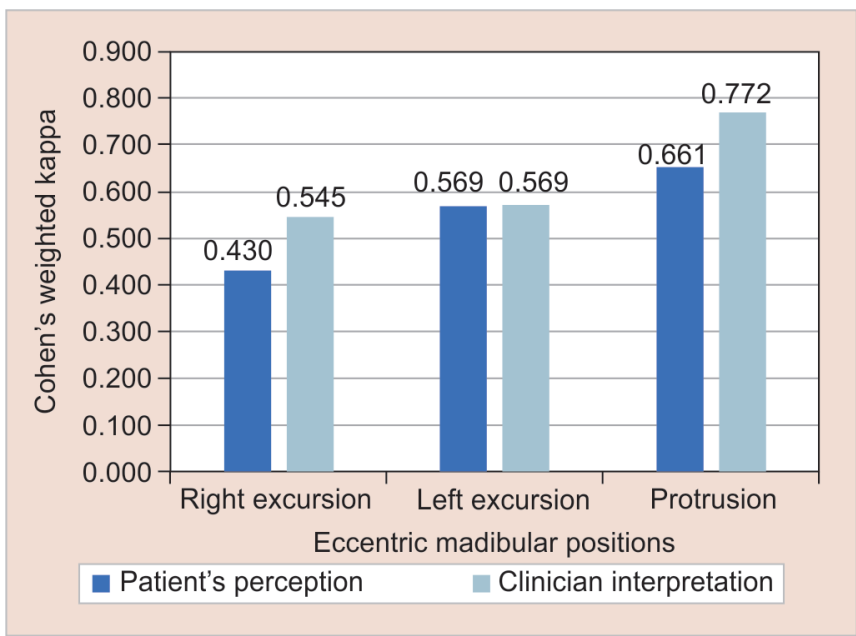

Fig. 2: Cohen's weighted kappa analysis between patient's perception, clinician interpretation, and T-Scan III analysis at eccentric positions 
Table 5: Cohen's weighted kappa of clinician reliability testing

\begin{tabular}{lll}
\hline Eccentric positions & Kappa score & Agreement level \\
\hline Right excursion & 0.546 & Moderate \\
Left excursion & 0.692 & Substantial \\
Protrusion & 0.895 & Almost perfect \\
\hline
\end{tabular}

of T-Scan III can be used up to 20 times, and data from the first 20 repeated bites were consistent and reliable. ${ }^{28}$ More importantly, the main advantage of T-Scan III is the elimination of several variabilities such as material properties and clinician's experience. ${ }^{29,30}$

Even though studies endorsed the benefits of T-Scan III in terms of high accuracy and reliability, ${ }^{10,28,31}$ the 100- $\mu$ m thick of the HD sensor was approximately 4-12 fold greater than the physiologic axial tooth movement. ${ }^{32}$ Consequently, it may affect patient's bite and cause bite deviation. To minimize these problems, dental students who can practically be trained to bite at MIP and perform jaw movement at different positions were recruited for the study. They were trained to bite on the desired position before data collection. Moreover, the U-shaped HD sensor had a uniform thickness. Therefore, all occluding teeth can simultaneously contact on both sides and the errors resulting from the thickness of the HD sensor were minimized. ${ }^{11}$ Additionally, the data collection was done three times and reported in consensus.

Even if clinical examination using traditional occlusal indicators may be the most convenient method to examine dental occlusion, no previous research assured the validity of clinician interpretation of dental occlusion in eccentric mandibular positions. Kerstein and Radke investigated the validity of dental occlusion examination evaluated by the clinician using articulating paper marks in relation to T-Scan III analysis. They found that dentists cannot determine the relative bite force effectively. ${ }^{33}$ In this study, clinician reliability testing (kappa score) revealed moderate to an almost perfect agreement even with traditional occlusal indicators. This finding maybe because of the restricted properties of the traditional occlusal indicators ${ }^{1}$ and the clinical experience of the examiner.

\section{ConCLUSION}

This is the first study to have evaluated the reliability of patient and clinician interpretation in an examination of dental occlusion in eccentric positions compared to the digital occlusal analyzer known as T-Scan III. The highest agreement with T-Scan III was protrusion followed by left and right excursion, respectively. The patient's perception was less reliable (less consistent with T-Scan III analysis) than clinician interpretation in protrusion and right excursion. However, both patients and clinicians equally perceived dental occlusion during the left excursion.

The limitation of this study was that there was only one dentist who evaluated dental occlusion and all participants were dental students who might not represent the patients in the other groups. Therefore, future studies should have a wide variety of dentists and patients. Moreover, a comparison of the combined patient and clinician interpretations with T-Scan III analysis may be beneficial.

\section{Acknowledgments}

The authors thank Dr Pornsuda Norchai for statistical analysis. This study was supported by the Graduate Students Research Funds, Faculty of Dentistry, Naresuan University.

\section{References}

1. Kerstein RB. Handbook of research on computerized occlusal analysis technology applications in dental medicine. Hershey: IGI Global; 2015.

2. Crum RJ, Loiselle R. Oral perception and proprioception: a review of the literature and its significance to prosthodontics. J Prosthet Dent 1972;28(2):215-230. DOI: 10.1016/0022-3913(72)90141-2.

3. Kobayashi M. Mechanisms of orofacial sensory processing in the rat insular cortex. J Oral Biosci 2018;60(3):59-64. DOI: 10.1016/j. job.2018.04.002.

4. Türker KS, Sowman PF, Tuncer M, et al. The role of periodontal mechanoreceptors in mastication. Arch Oral Biol 2007;52(4):361-364. DOI: 10.1016/j.archoralbio.2006.11.014.

5. Pfaffmann C. Afferent impulses from the teeth due to pressure and noxious stimulation. J Physiol 1939;97(2):207-219. DOI: 10.1113/ jphysiol.1939.sp003800.

6. Trulsson M. Force encoding by human periodontal mechanoreceptors during mastication. Arch Oral Biol 2007;52(4):357-360. DOI: 10.1016/j. archoralbio.2006.09.011.

7. Cash R, Linden R. The distribution of mechanoreceptors in the periodontal ligament of the mandibular canine tooth of the cat. J Physiol 1982;330(1):439-447. DOI: 10.1113/jphysiol.1982.sp014350.

8. Trulsson M, Johansson R. Encoding of amplitude and rate of forces applied to the teeth by human periodontal mechanoreceptive afferents. J Neurophysiol 1994;72(4):1734-1744. DOI: 10.1152/ jn.1994.72.4.1734.

9. Trulsson M, Johansson R, Olsson K. Directional sensitivity of human periodontal mechanoreceptive afferents to forces applied to the teeth. J Physiol 1992;447(1):373-389. DOI: 10.1113/jphysiol.1992. sp019007.

10. Cohen-Lévy J, Cohen N. Computerized occlusal analysis in dentofacial orthopedics: indications and clinical use the T-scan III system. J Dentofacial Anom Orthod 2012;15(2):203-227. DOI: 10.1051/ odfen/2012103.

11. Kim J. Computerized occlusion using T-Scan III. South Boston: MA Tekscan; 2014. pp. 1-35.

12. Kerstein RB. Time-sequencing and force-mapping with integrated electromyography to measure occlusal parameters. In: Daskalaki A, ed. Clinical Technologies: Concepts, Methodologies, Tools and Applications. Hershey: IGI Global; 2010. pp. 88-110.

13. Liu CW, Chang YM, Shen YF, et al. Using the T-Scan III system to analyze occlusal function in mandibular reconstruction patients: a pilot study. Biomed J 2015;38(1):52-57. DOI: 10.1016/j.bj.2018.02.002.

14. Di Berardino F, Filipponi $\mathrm{E}$, Schiappadori $\mathrm{M}$, et al. The occlusal imaging and analysis system by T-Scan III in tinnitus patients. Biomed J 2016;39(2):139-144. DOI: 10.1016/j.bj.2016.04.001.

15. Ruttitivapanich N, Tansalarak R, Palasuk J, et al. Correlation of bite force interpretation in maximal intercuspal position among patient, clinician, and T-Scan III system. Eur J Dent 2019;13(3):330-334. DOI: 10.1055/s-0039-1693755.

16. Landis JR, Koch GG. The measurement of observer agreement for categorical data. Biometrics 1977;33(1):159-174. DOI: 10.2307/2529310.

17. Siirilä HS, Laine $P$. The tactile sensibility of the parodontium to slight axial loadings of the teeth. Acta Odontol Scand 1963;21(5):415-429. DOI: 10.3109/00016356309028204.

18. Ross IF. Incisal guidance of natural teeth in adults. J Prosthet Dent 1974;31(2):155-162. DOI: 10.1016/0022-3913(74)90050-X.

19. Hannam A. The response of periodontal mechanoreceptors in the dog to controlled loading of the teeth. Arch Oral Biol 1969;14(7):781-791. DOI: 10.1016/0003-9969(69)90169-1.

20. Johnsen SE, Svensson KG, Trulsson M. Forces applied by anterior and posterior teeth and roles of periodontal afferents during hold-andsplit tasks in human participants. Exp Brain Res 2007;178(1):126-134. DOI: 10.1007/s00221-006-0719-9.

21. Trulsson M. Sensory-motor function of human periodontal mechanoreceptors. J Oral Rehabil 2006;33(4):262-273. DOI: 10.1111/j.1365-2842.2006.01629.x. 
22. Keith JF, Steven MM, Carl FD, et al. The glossary of prosthodontic terms. J Prosthet Dent 2017;117(5):1-105. DOI: 10.1016/j.prosdent.2016. 12.001.

23. Ogawa T, Ogimoto T, Koyano K. Pattern of occlusal contacts in lateral positions: canine protection and group function validity in classifying guidance patterns. J Prosthet Dent 1998;80(1):67-74. DOI: 10.1016/ S0022-3913(98)70093-9.

24. Shefter GJ, McFall JrWT. Occlusal relations and periodontal status in human adults. J Periodontol 1984;55(6):368-374. DOI: 10.1902/ jop.1984.55.6.368.

25. Beyron $\mathrm{H}$. Occlusal relations and mastication in Australian aborigines. Acta Odontol Scand 1964;22(6):597-678. DOI: 10.3109/00016356409058580.

26. Weinberg L. A cinematic study of centric and eccentric occlusions. J Prosthet Dent 1964;14(2):290-293. DOI: 10.1016/0022-3913(64) 90088-5.

27. Goldstein GR. The relationship of canine-protected occlusion to a periodontal index. J Prosthet Dent 1979;41(3):277-283. DOI: 10.1016/0022-3913(79)90007-6.
28. Kerstein RB, Lowe M, Harty M, et al. A force reproduction analysis of two recording sensors of a computerized occlusal analysis system. Cranio 2006;24(1):15-24. DOI: 10.1179/crn.2006.004.

29. Koos B, Godt A, Schille C, et al. Precision of an instrumentation-based method of analyzing occlusion and its resulting distribution of forces in the dental arch. J Orofac Orthop 2010;71(6):403-410. DOI: 10.1007/ s00056-010-1023-7.

30. Kerstein R. Articulating paper mark misconceptions and computerized occlusal analysis technology. Dent Implantol Update 2008;19(6):41-46.

31. Lila-Krasniqi ZD, Shala KS, Pustina-Krasniqi T, et al. Differences between centric relation and maximum intercuspation as possible cause for development of temporomandibular disorder analyzed with T-Scan III. Eur J Dent 2015;9(4):573-579. DOI: 10.4103/13057456.172627.

32. Hoffmann O, Zafiropoulos G. Tooth-implant connection: a review. J Oral Implantol 2012;38(2):194-200. DOI: 10.1563/AAIDJOI-D-10-00071.

33. Kerstein RB, Radke J. Clinician accuracy when subjectively interpreting articulating paper markings. Cranio 2014;32(1):13-23. DOI: $10.1179 / 0886963413 Z .0000000001$. 